\title{
Accretion of chemically fractionated material on a wide binary with a blue straggler ${ }^{\star}$
}

\author{
S. Desidera ${ }^{1}$, R. G. Gratton ${ }^{1}$, S. Lucatello ${ }^{1}$, M. Endl ${ }^{2}$, and S. Udry ${ }^{3}$ \\ ${ }^{1}$ INAF - Osservatorio Astronomico di Padova, Vicolo dell' Osservatorio 5, 35122 Padova, Italy \\ e-mail: silvano.desidera@oapd.inaf.it \\ 2 McDonald Observatory, The University of Texas at Austin, Austin, TX 78712, USA \\ 3 Observatoire de Geneve, 51 Ch. des Maillettes, 1290 Sauverny, Switzerland
}

Received 31 July 2006 / Accepted 2 October 2006

\begin{abstract}
Context. The components of the wide binary HIP $64030=$ HD 113984 show a large (about 0.25 dex) iron content difference (Desidera et al. 2006). The positions of the components on the color magnitude diagram suggest that the primary is a blue straggler. Aims. We studied the abundance difference of several elements besides iron, and we searched for stellar and substellar companions around the components to unveil the origin of the observed iron difference.

Methods. A line-by-line differential abundance analysis for several elements was performed for iron, while suitable spectral synthesis was performed for $\mathrm{C}, \mathrm{N}$, and Li. High precision radial velocities obtained with the iodine cell were combined with available literature data.

Results. The analysis of additional elements shows that the abundance difference for the elements studied increases with increasing condensation temperature, suggesting that accretion of chemically fractionated material might have occurred in the system. Alteration of $\mathrm{C}$ and $\mathrm{N}$ likely due to $\mathrm{CNO}$ processing is also observed. We also show that the primary is a spectroscopic binary with a period of 445 days and moderate eccentricity. The minimum mass of the companion is $0.17 M_{\odot}$.

Conclusions. Two scenarios were explored to explain the observed abundance pattern. In the first, all abundance anomalies arise on the blue straggler. If this is the case, the dust-gas separation may have been occurred in a circumbinary disk around the blue straggler and its expected white dwarf companion, as observed in several $R V$ Tauri and post AGB binaries. In the second scenario, accretion of dust-rich material occurred on the secondary. This would also explain the anomalous carbon isotopic ratio of the secondary. Such a scenario requires that a substantial amount of mass lost by the central binary has been accreted by the wide component. Further studies to compare the two scenarios are proposed.
\end{abstract}

Key words. stars: abundances - stars: blue stragglers - stars: individual: HIP 64030 - stars: binaries: visual stars: binaries: spectroscopic - techniques: spectroscopic

\section{Introduction}

In Desidera et al. $(2004,2006 \mathrm{c})$ we performed highprecision differential abundance analysis of 50 wide visual binaries with similar components. Only one pair (HIP $64030=$ HD $113984=$ ADS 8786) was shown to have an abundance difference as large as $0.27 \mathrm{dex}$, with the secondary being more metal rich. No other pair has an abundance difference larger than $0.09 \mathrm{dex}$, and in most cases differences are lower than 0.03 dex. This allowed us to conclude that the occurrence of large alterations of stellar abundances due to the ingestion of metal-rich material is not a common event, placing limits on the amount of accreted rocky material similar to estimates of the rocky material accreted by the Sun during its main sequence lifetime. The positions of the components of HIP 64030 on the color

$\star$ Based on observations collected at the European Southern Observatory, Chile, using FEROS spectrograph (proposal ID: 70.D-0081), on observations made with the Italian Telescopio Nazionale Galileo (TNG) operated on the island of La Palma by the Fundacion Galileo Galilei of the INAF (Istituto Nazionale di Astrofisica) at the Spanish Observatorio del Roque de los Muchachos of the Instituto de Astrofisica de Canarias, and on observations made at McDonald Observatory. magnitude diagram suggest that the primary is a blue straggler. Therefore, the abundance difference may be somewhat linked to the peculiar evolutionary history of the system.

Abundance anomalies are expected to occur in blue stragglers formed through mass transfer events (McCrea 1964 mechanism) or merging of WUMa binaries, while no abundance changes are expected in case of collisions (Shetrone \& Sandquist 2000). For a field blue straggler likely formed through mass transfer, abundance anomalies of light elements $(\mathrm{Li}, \mathrm{C}, \mathrm{N})$ are expected and, in the case of sufficiently massive AGB donor, selements enhancements are, too. The appearance of first dredgeup products in the atmosphere of an RGB donor would make the star $\mathrm{N}$ rich and slightly $\mathrm{C}$ poor, altering the ${ }^{12} \mathrm{C} /{ }^{13} \mathrm{C}$ isotopic ratio at the same time. Different patterns of carbon and neutron capture enhancements may arise depending on the mass and the metallicity of an AGB donor (see, e.g., Norris et al. 1997). Iron is not expected to be altered in these processes. Therefore, other mechanism(s) should play a fundamental role in the generation of the abundance pattern we observed in HIP 64030.

The study of several elements besides iron would allow a more complete picture of the abundance anomalies in this system and possibly help us to find a full explanation for them. Such 
a study is presented in this paper, together with a radial velocity (hereafter $R V$ ) monitoring of the components and a full description of the properties of the system that can be derived from the available information.

The outline of the paper is as follows: Sect. 2 presents the basic parameters of the system; in Sect. 3 we show that the blue straggler component is a spectroscopic binary and we derive clues on the orbit of the wide pair; Sect. 4 presents the procedures adopted to derive abundance of several elements; Sect. 5 presents the results of the differential abundance analysis, considering both the differential analysis between the components and the comparison with typical stars of similar metallicity and galactic population. In Sect. 6 we discuss possible scenarios to explain the observed abundance pattern. Finally, in Sect. 7 we summarize our conclusion and suggest future developments.

\section{Stellar parameters}

Table 1 lists the basic stellar properties of the components of the binary system HIP 64030. The pair is fairly metal poor. Our temperatures and gravities imply an anomalous position in the CMD (Fig. 1). The primary (brighter component) has properties compatible with an intermediate age (about $6 \mathrm{Gyr}$ ), while the secondary appears somewhat evolved, indicating an older age (8-12 Gyr). The details are sensitive to the adopted absolute magnitudes (i.e., distance). In this paper, we adopt the spectroscopic distance ( $66 \mathrm{pc})$ instead of that derived from the Hipparcos parallax (adopted in Desidera et al. 2006c) because of the fairly large error of the latter (about 15\%), the presence of a short period companion to the primary discussed in Sect. 3, that is not considered in the derivation of the parallax, and the better fit of the Balmer lines for the temperature resulting from the revised distance. The spectroscopic distance is about $1 \sigma$ shorter than the Hipparcos one. Kinematic parameters and galactic orbit were derived as in Barbieri \& Gratton (2000), considering the revised distance and averaging radial velocity (Sect. 3.1) and Hipparcos proper motion of the two components.

The enhancement of $\alpha$ elements (see Sect. 5) and the kinematics indicate that the pair is a likely member of the thick disk and thus is very old. This is fully consistent with the age of the secondary derived by isochrone fitting, 10-12 Gyr (Fig. 1). Therefore, the primary appears anomalous, being too bright for its age. It is thus very likely to be a blue straggler.

Stellar masses were derived using the $\alpha$-enhanced isochrones by Salasnich et al. (2000). Masses of $1.04 M_{\odot}{ }^{1}$ and $0.96 M_{\odot}$ and ages of about 6 and 10 Gyr for the primary and secondary, respectively, assuming the surface abundances of our analysis (Sect. 5). If only the convective zone of the secondary is enriched with heavy elements, the use of stellar models with the metallicity corresponding to the surface abundances is not appropriate. From the stellar models with polluted convective zones (Cody \& Sasselov 2005), it results that a larger metal content in the convective zone moves a star toward lower effective temperatures keeping the stellar luminosity nearly constant. The mass of the star with a polluted convective zone can be roughly derived from the isochrone with the chemical composition expected for the inner parts of the star, assuming the observed absolute magnitude and an effective temperature warmer than the observed one (by about $150 \mathrm{~K}$ for HIP 64030B, assuming a temperature shift similar to that derived by Cody \& Sasselov (2005) for a 0.2 dex abundance difference for HD 209458). The mass of HIP 64030B derived in this way results $0.91 M_{\odot}$. Conversely, if the primary

\footnotetext{
1 This is the mass of the visible component HIP 64030Aa, see Sect. 3.
}

Table 1. Stellar properties of the components of HIP 64030.

\begin{tabular}{|c|c|c|c|}
\hline Parameter & HIP 64030A & HIP 64030B & Ref \\
\hline$\mu_{\alpha}(\mathrm{mas} / \mathrm{yr})$ & $-101.92 \pm 1.66$ & $-97.60 \pm 2.36$ & 1 \\
\hline$\mu_{\delta}(\mathrm{mas} / \mathrm{yr})$ & $-105.92 \pm 1.40$ & $-102.24 \pm 2.20$ & 1 \\
\hline$R V\left(\mathrm{~km} \mathrm{~s}^{-1}\right)$ & $-90.1 \pm 0.2$ & $-89.6 \pm 0.2$ & 2 \\
\hline$\pi$ (mas) & \multicolumn{2}{|c|}{$13.24 \pm 1.99$} & 1 \\
\hline$d_{\text {spec }}(\mathrm{pc})$ & \multicolumn{2}{|c|}{$66 \pm 10$} & 2 \\
\hline$U\left(\mathrm{~km} \mathrm{~s}^{-1}\right)$ & \multicolumn{2}{|c|}{$-38.3 \pm 2.0$} & 2 \\
\hline$V\left(\mathrm{~km} \mathrm{~s}^{-1}\right)$ & \multicolumn{2}{|c|}{$-11.1 \pm 6.5$} & 2 \\
\hline$W\left(\mathrm{~km} \mathrm{~s}^{-1}\right)$ & \multicolumn{2}{|c|}{$-91.9 \pm 2.0$} & 2 \\
\hline$R_{\min }(\mathrm{kpc})$ & \multicolumn{2}{|c|}{$7.84 \pm 0.16$} & 2 \\
\hline$R_{\max }(\mathrm{kpc})$ & \multicolumn{2}{|c|}{$9.77 \pm 0.34$} & 2 \\
\hline$e$ & \multicolumn{2}{|c|}{0.11} & 2 \\
\hline$z_{\max }(\mathrm{kpc})$ & \multicolumn{2}{|c|}{$1.98 \pm 0.07$} & 2 \\
\hline$V$ & $7.528 \pm 0.004$ & $8.095 \pm 0.007$ & 3 \\
\hline$(B-V)$ & $0.439 \pm 0.013$ & $0.539 \pm 0.024$ & 3 \\
\hline$H_{\mathrm{p}}$ & $7.639 \pm 0.005$ & $8.219 \pm 0.008$ & 1 \\
\hline$H_{\mathrm{p}}$ scatter & \multicolumn{2}{|c|}{$0.010^{a}$} & 1 \\
\hline$J$ & $6.596 \pm 0.018$ & $6.961 \pm 0.020$ & 4 \\
\hline$H$ & $6.409 \pm 0.044$ & $6.703 \pm 0.033$ & 4 \\
\hline$K$ & $6.325 \pm 0.017$ & $6.610 \pm 0.023$ & 4 \\
\hline$b-y$ & \multicolumn{2}{|c|}{$0.332^{a} \pm 0.004$} & 5 \\
\hline$m_{1}$ & \multicolumn{2}{|c|}{$0.126^{a} \pm 0.005$} & 5 \\
\hline$c_{1}$ & \multicolumn{2}{|c|}{$0.398^{a} \pm 0.006$} & 5 \\
\hline ST & F5V & F9V & 6 \\
\hline$M_{V}$ & $3.47 \pm 0.25$ & $4.04 \pm 0.25$ & 2 \\
\hline$T_{\text {eff }}(\mathrm{K})$ & $6283 \pm 100$ & $5834 \pm 100$ & 2 \\
\hline$\Delta T_{\text {eff }}(\mathrm{K})$ & \multicolumn{2}{|c|}{$451 \pm 20$} & 2 \\
\hline $\log g$ & $4.07 \pm 0.15$ & $4.09 \pm 0.15$ & 2 \\
\hline $\log R_{\mathrm{HK}}^{\prime}$ & -5.06 & -5.12 & 7 \\
\hline$v \sin i\left(\mathrm{~km} \mathrm{~s}^{-1}\right)$ & 4.1 & 2.5 & 7 \\
\hline$L_{\mathrm{X}}(\mathrm{erg} / \mathrm{s})$ & \multicolumn{2}{|c|}{$<1.0 \times 10^{29 a}$} & 2 \\
\hline$[\mathrm{Fe} / \mathrm{H}]$ & $-0.57 \pm 0.10$ & $-0.32 \pm 0.10$ & 2 \\
\hline$\Delta[\mathrm{Fe} / \mathrm{H}]$ & \multicolumn{2}{|c|}{$-0.251 \pm 0.020$} & 2 \\
\hline $\operatorname{Mass}\left(M_{\odot}\right)$ & $\begin{array}{c}1.04 \pm 0.05^{b} \\
>1.21^{c}\end{array}$ & $0.91 \pm 0.05$ & $\begin{array}{l}2 \\
2\end{array}$ \\
\hline Age (Gyr) (isoc.) & $\sim 6$ & $8-12$ & 2 \\
\hline Age $(\mathrm{Gyr})\left(R_{\mathrm{HK}}\right)$ & 7 & 8 & 7 \\
\hline
\end{tabular}

References: 1: Hipparcos (ESA 1997); 2: This paper; 3: Desidera et al. (2006c); 4: 2MASS (Cutri et al. 2003); 5: Olsen (1998); 6: Abt (1981); 7: Desidera et al. (2006b).

${ }^{a} \mathrm{~A}+\mathrm{B} ;{ }^{b} \mathrm{Aa} ;{ }^{c} \mathrm{Aa}+\mathrm{Ab}$.

has a stellar atmosphere more metal poor than the stellar interior, its actual mass would be slightly larger than $1.04 M_{\odot}$. In the following, we will assume $M_{A a}=1.04 M_{\odot}$ and $M_{B}=0.91 M_{\odot}$, taking into account that the impact of the different mass assumptions on the results of this paper are minor.

Blue stragglers and ultra-lithium depleted stars often show moderate rotational velocity (Fuhrmann \& Bernkopf 1999; Ryan et al. 2002). This is thought to arise because of the acquisition of angular momentum during the mass transfer process. In HIP 64030, the BS component shows a slightly enhanced $v \sin i\left(4.1 \mathrm{~km} \mathrm{~s}^{-1}\right)$ with respect to the secondary, a slow rotator as expected for a very old $\operatorname{star}\left(v \sin i=2.5 \mathrm{~km} \mathrm{~s}^{-1}\right)^{2}$. The low

2 These values are calculated for the $B-V$ color obtained by Tycho. Adopting the colors corresponding to the spectroscopic temperatures increases the rotational velocities by just $0.2 \mathrm{~km} \mathrm{~s}^{-1}$ because of the smaller macroturbulent velocity. 

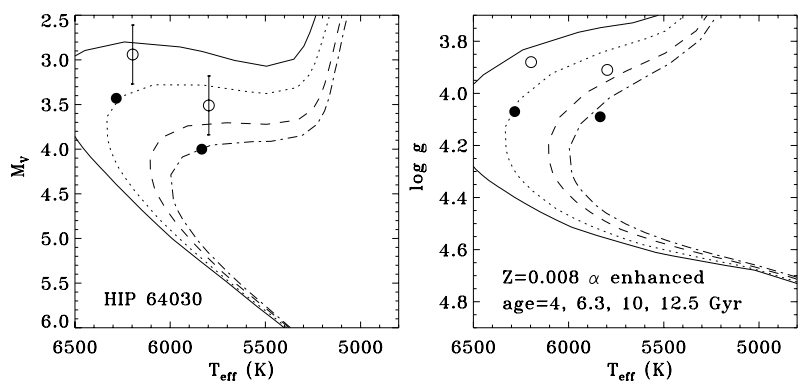

Fig. 1. Position of the components of HIP 64030 in the HR diagram (left panel) and in the $T_{\mathrm{eff}}-\log g$ diagram (right panel). Open circles: results of abundance analysis performed adopting the Hipparcos distance; filled circles: adopting spectroscopic distances. Isochrones by Salasnich et al. (2000) with $Z=0.008$ and $[\alpha / \mathrm{Fe}]=0.3$, close to the surface abundances of the primary, are overplotted. It is not possible to fit both components with a single isochrone. The most likely explanation is that the primary is a blue straggler.

chromospheric emission, the lack of X-ray detection by ROSAT (Voges et al. 2000), and the small photometric variability from Hipparcos are further indications of a fairly low rotation rate.

\section{Binarity}

The binary fraction among field blue stragglers is very high (Preston \& Sneden 2000; Carney et al. 2005). The lack of double-lined spectroscopic binaries and the distribution of the mass function suggest that most of the companion are white dwarfs with masses of about $0.55 M_{\odot}$. The period and eccentricity distributions are also different with respect to a comparison sample of normal main sequence stars (Duquennoy \& Mayor 1991). Most of the blue stragglers have periods in the range 100 to 3000 days and eccentricities smaller than those of field binaries. The low eccentricity can be explained with the occurrence of angular momentum dissipation as part of the mass transfer process (e.g., McClure 1997). All these properties are expected if these objects were formed predominately via the McCrea (1964) scenario. If HIP 64030A is indeed a blue straggler, as we are claiming, we then expect it has a white dwarf companion with short orbital period and low eccentricity. In this section we consider the evidence in favor of this hypothesis that results from radial velocities and astrometric measurement, and the clues on the orbit of the wide pair.

\subsection{Radial velocity}

The available $R V$ measurements taken with traditional techniques (errors larger than $0.2 \mathrm{~km} \mathrm{~s}^{-1}$ ), including previously unpublished CORAVEL data, suggest the occurrence of low-amplitude radial velocity variations for HIP 64030A (Table 2). Conclusive evidence of the radial velocity variability comes from ten spectra taken with SARG, the high resolution spectrograph of the TNG (Gratton et al. 2001), and at the Harlan J. Smith $2.7 \mathrm{~m}$ telescope at McDonald Observatory. These spectra were acquired with the iodine cell and analyzed as those for the on-going planet search programs using these instruments (Desidera et al. 2006a; Endl et al. 2000; Wittenmyer et al. 2006). Errors in differential radial velocities are about $10 \mathrm{~m} / \mathrm{s}$ and $15 \mathrm{~m} / \mathrm{s}$ for SARG and McDonald, respectively, slightly larger than is typical for stars of similar magnitude observed with these instruments because of the shallowness of the spectral lines of this
Table 2. Radial velocity of the components of HIP 64030.

\begin{tabular}{cccl}
\hline \hline Epoch & $R V(\mathrm{~A})$ & $R V(\mathrm{~B})$ & Ref. \\
\hline$<34379$ & $-92.2 \pm 1.9$ & $-87.0 \pm 1.5$ & 1 \\
34817.50 & $-88.3 \pm 0.8^{a}$ & $-89.2 \pm 0.9^{a}$ & 2 \\
35856.50 & & $-90.0 \pm 0.6^{a}$ & 2 \\
35937.50 & $-90.7 \pm 0.9^{a}$ & & 2 \\
36266.50 & $-91.2 \pm 0.7^{a}$ & $-90.4 \pm 0.8^{a}$ & 2 \\
43912.695 & $-92.53 \pm 0.53$ & $-89.51 \pm 0.48$ & 3 \\
44314.588 & $-92.67 \pm 0.56$ & $-90.42 \pm 0.47$ & 3 \\
44388.62 & $-90.4 \pm 1.4$ & $-89.1 \pm 1.4$ & 4 \\
44636.696 & $-89.00 \pm 0.52$ & $-89.13 \pm 0.39$ & 3 \\
49777.535 & $-92.08 \pm 0.54$ & $-89.62 \pm 0.48$ & 3 \\
52721.16 & $-91.8 \pm 0.2$ & $-89.6 \pm 0.2$ & 5 \\
53483.61 & $-84.999 \pm 0.008^{b}$ & $-89.625 \pm 0.004^{b}$ & 6 \\
53514.43 & $-86.817 \pm 0.006^{b}$ & $-89.622 \pm 0.004^{b}$ & 6 \\
53566.70 & $-89.746 \pm 0.015^{b}$ & & 7 \\
53780.78 & $-92.372 \pm 0.012^{b}$ & & 6 \\
53808.88 & $-91.205 \pm 0.018^{b}$ & & 7 \\
53813.70 & $-91.138 \pm 0.009^{b}$ & $-89.629 \pm 0.009^{b}$ & 6 \\
53864.82 & $-87.288 \pm 0.017^{b}$ & & 7 \\
53871.52 & $-86.857 \pm 0.006^{b}$ & $-89.614 \pm 0.004^{b}$ & 6 \\
53872.51 & $-86.769 \pm 0.014^{b}$ & $-89.607 \pm 0.004^{b}$ & 6 \\
53910.72 & $-84.485 \pm 0.014^{b}$ & & 7 \\
\hline
\end{tabular}

References: 1: Wilson (1953); 2: Struve \& Zebergs (1959); 3: CORAVEL (this paper); 4: Andersen \& Nordstrom (1985); 5: FEROS (Desidera et al. 2006b); 6: SARG (this paper); 7: McDonald (this paper).

${ }^{a}$ Internal errors are quoted in the original reference. Comparison with Nordstrom et al. (2004) for stars in common that are not spectroscopic binaries indicates that the $R V$ zero point is the same and that a further $0.5 \mathrm{~km} \mathrm{~s}^{-1}$ error should be present. Such a error was added in quadrature to the internal errors.

${ }^{b}$ Internal errors of differential radial velocities obtained using iodine lines. Errors on absolute radial velocities are about $0.2-0.3 \mathrm{~km} \mathrm{~s}^{-1}$.

F5 metal-poor star. The differential radial velocities measured with the iodine cell technique were placed on an absolute scale to allow a combination of the whole data set by cross-correlation using the spectral orders between 4700 and $4970 \AA$ that are free of iodine lines, further adjusting the zero-point of the McDonald spectra using telluric lines.

The highest peak of the Scargle-Lomb periodogram of the radial velocities is at 445 days. A Keplerian fitting yields the orbital parameters listed in Table 3. The radial velocities phased to this orbital period are shown in Fig. 2. A Keplerian fitting for other possible periods resulting from the periodogram clearly indicates that the 445 days period is the real one.

The minimum mass of the companion is about $0.17 M_{\odot}$. This is compatible with the presence of a white dwarf companion, as expected for blue stragglers formed through mass transfer from an RGB companion. The orbital period and eccentricity are also normal for a field blue straggler (Preston \& Sneden 2000). The secondary instead shows a constant $R V$, fully compatible with the observational errors, both on decade timescales with errors of about $1 \mathrm{~km} \mathrm{~s}^{-1}$ and on the two-year timespan of SARG observations with errors of about $10 \mathrm{~m} / \mathrm{s}$. Hereafter we refer to HIP 64030Aa as the current primary star (the blue straggler), to HIP 64030Ab as the unseen spectroscopic companion (which we assume is a low mass white dwarf), and to HIP 64030B as the wide visual companion. 
Table 3. Preliminary orbital solution for HIP 64030A.

\begin{tabular}{lc}
\hline \hline Parameter & Value \\
\hline Period (d) & $445.8 \pm 0.8$ \\
$R V$ Semi-amplitude $\left(\mathrm{km} \mathrm{s}^{-1}\right)$ & $4.25 \pm 0.08$ \\
Eccentricity & $0.29 \pm 0.01$ \\
Longitude of periastron $(\mathrm{deg})$ & $342.5 \pm 2.3$ \\
Periastron passage $(\mathrm{JD}-2400000)$ & $43648 \pm 18$ \\
$\gamma\left(\mathrm{km} \mathrm{s}^{-1}\right)$ & $-90.06 \pm 0.07$ \\
$R V$ offset $(\mathrm{McDonald}-\mathrm{SARG})\left(\mathrm{km} \mathrm{s}^{-1}\right)$ & $-0.158 \pm 0.010$ \\
$R V$ offset $(\mathrm{CORAVEL}-\mathrm{SARG})\left(\mathrm{km} \mathrm{s}^{-1}\right)$ & $-0.26 \pm 0.30$ \\
Mass function $\left(M_{\odot}\right)$ & $0.00311 \pm 0.00018$ \\
& \\
$a(\mathrm{AU})$ & $1.215 \pm 0.002$ \\
$m \sin i\left(M_{\odot}\right)$ & $0.165 \pm 0.004$ \\
\hline
\end{tabular}

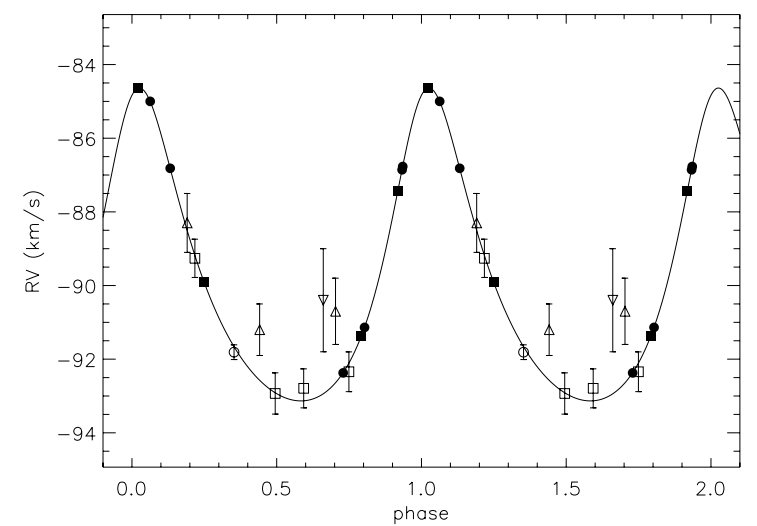

Fig. 2. The radial velocities of HIP 64030A phased to the preliminary orbital period (445 days). The best-fit orbital solution is overplotted. Radial velocities from different sources are represented by different symbols: filled circles: SARG (this paper); filled squares: McDonald (this paper); open circle: FEROS (Desidera et al. 2006b); empty squares: CORAVEL (this paper); empty upper triangles: Struve \& Zebergs (1959); upside down triangle: Andersen \& Nordstrom (1985).

\subsection{Astrometry}

Relative astrometry of the components allows us to place constraints on the orbital motion of the wide pair. Furthermore, it provides additional evidence of the binarity of the primary. Hipparcos detected relative motion between HIP 64030A and $\mathrm{B}(\mathrm{d} \rho / \mathrm{d} t=-0.005$ arcsec $/ \mathrm{yr}$ and $\mathrm{d} \theta / \mathrm{d} t=-0.030 \mathrm{deg} / \mathrm{yr})$. The literature position measurements (Table 4) are consistent with Hipparcos results concerning position angle, while the Hipparcos separation gradient is too steep to fit the old historical data reported by Aitken (1932), as shown in Fig. 3.

Part of the relative motion between $\mathrm{A}$ and $\mathrm{B}$ measured by Hipparcos is probably due to the contribution of HIP 64030Ab. The impact of this on the system parallax as derived by Hipparcos is difficult to assess. In some cases of unrecognized multiplicity, large parallax errors were discovered (see, e.g., Söderhjelm 1999).

The long-term relative motion of the wide binary coupled with the $R V$ difference can be used to constrain the orbit (Hauser \& Marcy 1999; Desidera et al. 2003). Assuming a $0.4 M_{\odot}$ companion for the spectroscopic companion of the blue straggler, a broad range of orbital parameters is compatible with the data (Fig. 4). Orbits with periastron down to about $100 \mathrm{AU}$ are possible.
Table 4. Relative position of the components of HIP 64030.

\begin{tabular}{cccc}
\hline \hline Epoch & $\rho$ & $\theta$ & Ref. \\
\hline 1830.01 & 7.24 & 3.1 & 1 \\
1842.68 & 7.40 & 4.2 & 1 \\
1910.33 & 7.23 & 1.9 & 1 \\
1915.68 & 7.28 & 1.3 & 1 \\
1923.62 & 7.52 & 0.9 & 1 \\
1950.38 & 7.06 & 0.5 & 2 \\
1955.27 & 7.12 & 0.2 & 3 \\
1956.24 & 7.12 & 0.4 & 3 \\
1979.35 & 7.24 & 358.8 & 4 \\
1982.35 & 6.96 & 356.4 & 5 \\
1991.25 & 7.013 & 359.0 & 6 \\
1992.50 & 7.03 & 358.91 & 7 \\
1997.36 & 6.88 & 358.7 & 8 \\
1997.37 & 7.23 & 359.8 & 8 \\
2000.19 & 6.99 & 358.8 & 9 \\
\hline
\end{tabular}

References: 1: Aitken (1932); 2: Muller (1950); 3: Gasteyer \& Eichhorn, (1964); 4: Heintz (1980); 5: Pannunzio \& Morbidelli (1983); 6: Hipparcos (ESA 1997); 7: Van Dessel \& Sinachopoulos (1993); 8: Alzner (1998); 9: 2MASS (Cutri et al. 2003).

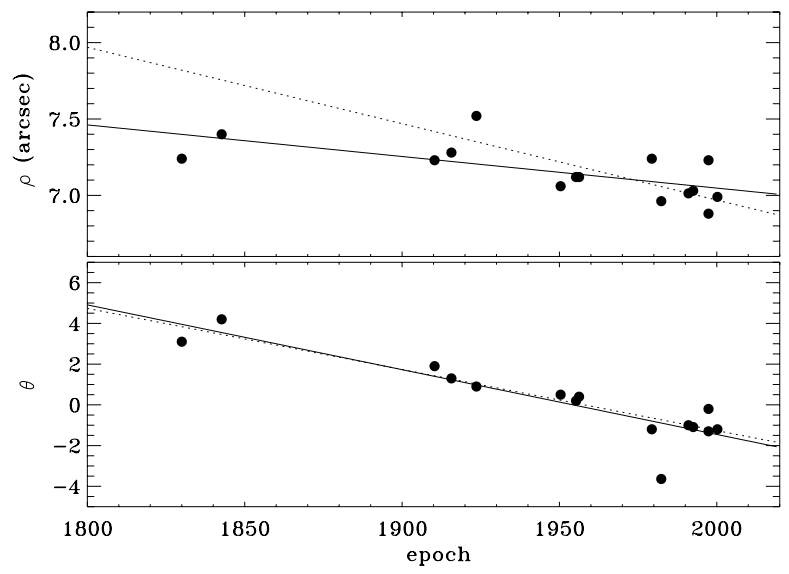

Fig. 3. Relative motion of HIP 64030A and B. The continuous line is the linear fitting to the data (without weighting). The dashed line is the relative motion measured by Hipparcos (extrapolated to a timescale much larger than the mission lifetime).

\section{Abundance analysis}

\subsection{Atmospheric parameters and iron abundance}

The binary system HIP 64030 shows a large abundance difference with the secondary being significantly more metal rich (Desidera et al. 2006c). The analysis performed adopting the spectroscopic distance yields the following atmospheric parameters: for the primary $T_{\text {eff }}=6283 \mathrm{~K}, \log g=4.07,[\mathrm{~A} / \mathrm{H}]=-0.57$, microturbulent velocity $=1.71 \mathrm{~km} \mathrm{~s}^{-1}$; for the secondary $T_{\mathrm{eff}}=$ $5834 \mathrm{~K}, \log g=4.09,[\mathrm{~A} / \mathrm{H}]=-0.32$, microturbulent velocity $=1.16 \mathrm{~km} \mathrm{~s}^{-1}$. The effective temperatures are warmer (by about $90 \mathrm{~K}$ ) and gravities higher (by about $0.15 \mathrm{dex}$ ) than the analysis presented in Desidera et al. (2006c) because of the fainter absolute magnitude adopted in the analysis. The stellar masses derived in Sect. 2 were used to derive stellar gravities. The iron abundance difference results -0.251 dex. Performing the analysis with different assumptions (different absolute magnitude, optimization of microturbulence instead of using a $\xi-T_{\text {eff }}$ relation, using $\mathrm{B}$ as reference, exclusion of vanadium lines to derive effective temperature) indicates that errors on this difference 



Fig. 4. Possible orbital parameters of the wide binary as a function of the (unknown) separation along the line of sight.

should not exceed 0.04 dex. The difference of effective temperature based on different techniques agree within errors. An error in temperature difference as large as $300 \mathrm{~K}$ would be required to eliminate the abundance difference.

The possibility that blending causes a spourious abundance difference also does not seem viable. Even if the primary is composed of a pair of equal components (i.e., equal spectrum with magnitude difference of $0.75 \mathrm{mag}$ ), the abundance difference and the anomalous position on the HR diagram could not be eliminated. A blend of the primary with a red star would make the age difference between the primary and the secondary even worse. In any case, there is no indication of contributions by the faint companion HIP 64040Ab on our spectra (asymmetries of line profiles, trends with wavelength of the observed abundance difference).

\subsection{Analysis of other elements}

The wide spectral coverage of the FEROS spectrograph allows the inclusion of spectral lines of several elements besides iron. The measurement of elements other than iron is useful to better constrain the properties of the stars and the origin of the observed iron difference.

\subsubsection{Analysis based on EW measurement}

Standard analysis based on EW measurement was performed for several elements. Hyperfine structure is taken into account for Sc, V, Mn, and Ba (see Gratton et al. 2003). Differential line-byline analysis was performed as for iron (Desidera et al. 2006c).

\subsubsection{Spectral synthesis of light elements}

From the synthesis of different spectral ranges, we obtained measurements for the abundances of $\mathrm{Li}, \mathrm{C}, \mathrm{N}$, and $\mathrm{O}$, as well as estimates on the $\mathrm{C}$ isotopic ratio. The line lists for the syntheses have been built as described in Lucatello et al. (2003), starting from the Kurucz line lists applying small wavelength and $\log g f$ adjustments to reproduce the solar spectrum. $\mathrm{C}$ abundance and $\mathrm{C}$ isotopic ratios were measured from several $\mathrm{CH}$ lines in the $G$ band around $4300 \AA$. An example of the features fitted is given in Fig. 5. The $\mathrm{C}$ abundance results $[\mathrm{C} / \mathrm{Fe}]=+0.20 \pm 0.10$ and $[\mathrm{C} / \mathrm{Fe}]=-0.10 \pm 0.10$ for the primary and the secondary,

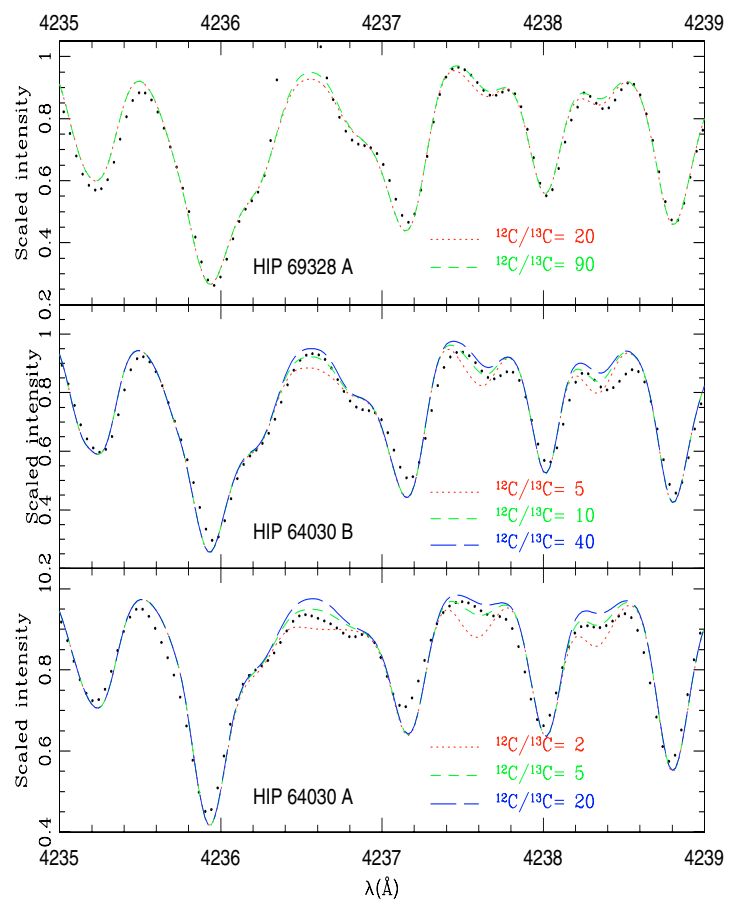

Fig. 5. A portion of the spectra used to derive the $\mathrm{C}$ isotopic ratio using spectral synthesis. Upper panel: the comparison star HIP 69328A, central panel: HIP 64030B, lower panel: HIP 64030A.



Fig. 6. CN spectral synthesis. Upper panel: HIP 64030B, lower panel: HIP 64030A.

respectively. The $\mathrm{C}$ isotopic ratio results ${ }^{12} \mathrm{C} /{ }^{13} \mathrm{C}=5-10 \pm 5$ for the primary and ${ }^{12} \mathrm{C} /{ }^{13} \mathrm{C}=30-40 \pm 10$ for the secondary. While the low isotopic ratio is expected for a blue straggler (see Sect. 5), it is unexpected for the secondary. We checked our synthesis procedure using a spectrum taken on the same run of the star HIP 69328A, which has atmospheric parameters fairly similar to HIP 64030B (Desidera et al. 2006c). A ${ }^{12} \mathrm{C} /{ }^{13} \mathrm{C}$ of $30-40$ is clearly excluded for this star (Fig. 5, upper panel).

The UV CN system at $3860 \AA$ was used to derive $N$ abundance (Fig. 6). The $N$ abundance results $[\mathrm{N} / \mathrm{Fe}]=+0.50 \pm 0.15$ and $[\mathrm{N} / \mathrm{Fe}]=-0.10 \pm 0.15$ for the primary and secondary, respectively. The $\mathrm{O}$ abundance was measured by synthesizing the $\lambda 6300.31 \AA$ forbidden line. To account for the contribution 
Table 5. Sensitivity of abundance differences to atmospheric parameters.

\begin{tabular}{|c|c|c|c|c|c|c|c|c|}
\hline Element & $\begin{array}{c}\Delta(\mathrm{A}-\mathrm{B}) \\
\Delta T_{\mathrm{eff}} \\
+100 \mathrm{~K}\end{array}$ & $\begin{array}{c}\Delta(\mathrm{A}-\mathrm{B}) \\
\Delta T_{\mathrm{eff}} \\
-100 \mathrm{~K}\end{array}$ & $\begin{array}{c}\Delta(\mathrm{A}-\mathrm{B}) \\
\Delta \log g \\
+0.10 \mathrm{dex}\end{array}$ & $\begin{array}{c}\Delta(\mathrm{A}-\mathrm{B}) \\
\Delta \log g \\
-0.10 \mathrm{dex}\end{array}$ & $\begin{array}{c}\Delta(\mathrm{A}-\mathrm{B}) \\
\Delta[\mathrm{A} / \mathrm{H}] \\
+0.10 \mathrm{dex}\end{array}$ & $\begin{array}{c}\Delta(\mathrm{A}-\mathrm{B}) \\
\Delta[\mathrm{A} / \mathrm{H}] \\
-0.10 \mathrm{dex}\end{array}$ & $\begin{array}{c}\Delta(\mathrm{A}-\mathrm{B}) \\
\Delta \xi \\
+0.20 \mathrm{~km} \mathrm{~s}^{-1}\end{array}$ & $\begin{array}{c}\Delta(\mathrm{A}-\mathrm{B}) \\
\Delta \xi \\
-0.20 \mathrm{~km} \mathrm{~s}^{-1}\end{array}$ \\
\hline $\mathrm{Fe} \mathrm{I}$ & -0.0810 & 0.0654 & -0.0103 & -0.0101 & -0.0037 & 0.0010 & 0.0278 & -0.0553 \\
\hline $\mathrm{Fe}$ II & 0.0265 & -0.0152 & -0.0328 & 0.0445 & -0.0145 & 0.0271 & 0.0574 & -0.0383 \\
\hline C I & 0.0544 & -0.0593 & -0.0346 & 0.0324 & 0.0044 & -0.0060 & 0.0010 & -0.0020 \\
\hline $\mathrm{Na} \mathrm{I}$ & -0.0485 & 0.0530 & 0.0065 & -0.0060 & -0.0015 & 0.0035 & 0.0110 & -0.0105 \\
\hline Mg I & -0.0400 & 0.0434 & 0.0044 & -0.0026 & -0.0006 & 0.0004 & 0.0084 & -0.0066 \\
\hline Al I & -0.0420 & 0.0440 & 0.0005 & -0.0020 & -0.0010 & -0.0010 & 0.0035 & -0.0055 \\
\hline Si I & -0.0296 & 0.0294 & 0.0005 & 0.0025 & -0.0056 & 0.0075 & 0.0117 & -0.0065 \\
\hline Si II & 0.0735 & -0.0795 & -0.0265 & 0.0285 & -0.0045 & 0.0080 & 0.0135 & -0.0075 \\
\hline S I & 0.0780 & -0.0543 & -0.0357 & 0.0333 & 0.0013 & -0.0023 & 0.0043 & -0.0053 \\
\hline $\mathrm{Ca} \mathrm{I}$ & -0.0727 & 0.0711 & 0.0173 & -0.0153 & -0.0027 & 0.0034 & 0.0397 & -0.0440 \\
\hline Ca II & 0.0527 & -0.0523 & -0.0247 & 0.0490 & 0.0007 & -0.0037 & -0.0023 & -0.0003 \\
\hline Sc II & -0.0004 & 0.0102 & -0.0335 & 0.0472 & -0.0190 & 0.0333 & 0.0531 & -0.0143 \\
\hline Ti I & -0.1059 & 0.1051 & 0.0053 & -0.0118 & -0.0006 & 0.0001 & 0.0434 & -0.0477 \\
\hline Ti II & 0.0115 & 0.0073 & -0.0332 & 0.0478 & -0.0163 & 0.0299 & 0.0493 & -0.0474 \\
\hline V I & -0.1670 & 0.1065 & 0.0011 & -0.0006 & -0.0002 & 0.0011 & 0.0124 & -0.0124 \\
\hline V II & 0.0135 & -0.0035 & -0.0335 & 0.0460 & -0.0115 & 0.0245 & 0.0215 & -0.0080 \\
\hline Cr I & -0.0850 & 0.0845 & 0.0050 & -0.0039 & 0.0001 & 0.0003 & 0.0442 & -0.0532 \\
\hline Cr II & 0.0247 & -0.0275 & -0.0334 & 0.0429 & -0.0126 & 0.0234 & 0.0437 & -0.0326 \\
\hline Co I & -0.0973 & 0.1269 & -0.0017 & 0.0030 & -0.0383 & 0.0040 & -0.0207 & -0.0787 \\
\hline $\mathrm{Ni} I$ & -0.0542 & 0.0621 & 0.0006 & 0.0026 & -0.0026 & 0.0051 & 0.0205 & -0.0157 \\
\hline $\mathrm{Cu} \mathrm{I}$ & -0.0140 & 0.0530 & -0.0030 & 0.0427 & -0.0045 & 0.0457 & 0.0135 & 0.0247 \\
\hline Zn I & 0.0756 & 0.0260 & -0.0074 & 0.0146 & -0.0137 & 0.0216 & 0.0600 & 0.0550 \\
\hline Sr I & -0.0077 & 0.0230 & -0.0327 & 0.0490 & -0.0190 & 0.0363 & -0.0295 & -0.0790 \\
\hline Y II & -0.1010 & 0.1080 & 0.0020 & 0.0000 & -0.0010 & 0.0020 & 0.0110 & -0.0110 \\
\hline $\mathrm{Zr}$ II & -0.0020 & 0.0120 & -0.0350 & 0.0490 & -0.0200 & 0.0330 & 0.0120 & 0.0020 \\
\hline Ba II & -0.0260 & 0.0300 & -0.0320 & 0.0430 & -0.0200 & 0.0300 & 0.1800 & -0.1950 \\
\hline La II & -0.0180 & 0.0320 & -0.0340 & 0.0510 & -0.0210 & 0.0400 & 0.0190 & -0.0030 \\
\hline Ce II & -0.0180 & 0.0320 & -0.0330 & 0.0496 & -0.0200 & 0.0390 & 0.0226 & -0.0100 \\
\hline Nd II & -0.0206 & 0.0354 & -0.0323 & 0.0500 & -0.0210 & 0.0407 & 0.0197 & -0.0030 \\
\hline
\end{tabular}

of the telluric lines to the spectra, we subtracted a properly scaled early-type, fast rotating star spectrum taken during the same observing run. The $\mathrm{O}$ abundance results $[\mathrm{O} / \mathrm{Fe}] \leq+0.40$ and $[\mathrm{O} / \mathrm{Fe}]=+0.20 \pm 0.15$ for the primary and secondary, respectively. It should be noted that the subtraction of the telluric lines prevents the accurate determination of abundance for $\mathrm{O}$ we would expect from spectra of the quality of the present data. $\mathrm{Li}$ was measured from the $6707 \AA$ resonance line (Fig. 7); however, in the case of HIP 64030A we could only derive an upper limit to it $(\mathrm{A}(\mathrm{Li})<1.8)$. For the secondary we found $\mathrm{A}(\mathrm{Li})=2.7 \pm 0.1$.

\subsection{Sensitivity of abundance differences to atmospheric parameters}

Table 5 shows the sensitivity of the abundance difference of the elements included in our analysis to variations of atmospheric parameters. The values were obtained by changing the atmospheric parameters of the secondary one by one. Different species have quite different sensitivities, indicating that it is not possible to erase the observed abundance difference for all the species changing the atmospheric parameters.

\section{Abundances of HIP $64030 A$ and $B$}

\subsection{Relative abundances with respect to iron}

As we will discuss in Sect. 6, it is not easy to understand which of the components can be considered the unpolluted one. The relative abundance with respect to iron may help for this and for a better classification of galactic population of our target.
Table 6 reports the results. Both components show enhancements of $\alpha$ elements $(\mathrm{Mg}, \mathrm{Si}, \mathrm{Ca})$. This supports the kinematic association to the thick disk population (and then the very old age for the system and the blue straggler status for the primary).

Nitrogen is strongly enhanced and the ${ }^{12} \mathrm{C} /{ }^{13} \mathrm{C}$ isotopic ratio is very low in the primary. We think these are the signatures of mass transfer from material processed through the CNO cycle within the companion, and dredged up on the surface during RGB phase. However, CNO processing should also produce some $\mathrm{C}$ depletion, which is not observed. The low lithium content of the primary is typical of blue stragglers (Ryan et al. 2001; Carney et al. 2005), probably because complete lithium depletion occurred in the atmosphere of the RGB/AGB donor.

The secondary has a more normal abundance pattern. However, the ${ }^{12} \mathrm{C} /{ }^{13} \mathrm{C}$ isotopic ratio is lower with respect to typical unevolved stars (Gratton et al. 2000). The lithium content of the secondary is similar to that of Hyades star and slightly larger than that of Li-rich stars in the open cluster M 67 of similar temperature (Fig. 8). This is somewhat unexpected. It suggests that no significant lithium depletion occurred during the main sequence lifetime of this star. Neutron capture elements are normal for both components. This indicates that the mass transfer event should have occurred during the RGB phase of the originally most massive star or during the AGB phase of a star not massive enough to produce s-elements enhancements.

\subsubsection{Differential analysis between the components}

The differential analysis provides evidence not only of the abundance anomalies caused by the accretion of $\mathrm{CNO}$ processed 
Table 6. Abundance difference between the components of HIP 64030.

\begin{tabular}{|c|c|c|c|c|c|c|c|c|c|}
\hline Element & {$[x / \mathrm{Fe}](\mathrm{A})$} & $\mathrm{rms}$ & {$[x / \mathrm{Fe}](\mathrm{B})$} & rms & $N_{\text {lines }}$ & $\Delta$ & $\mathrm{rms}$ & err & Remarks \\
\hline $\mathrm{Fe} \mathrm{I}$ & $-0.57^{1}$ & 0.113 & $-0.32^{1}$ & 0.093 & 105 & -0.251 & 0.071 & 0.007 & \\
\hline Fe II & $-0.57^{1}$ & 0.114 & $-0.33^{1}$ & 0.110 & 35 & -0.242 & 0.062 & 0.010 & \\
\hline C I & 0.41 & 0.188 & 0.10 & 0.039 & 3 & 0.053 & 0.217 & 0.125 & \\
\hline C & 0.20 & & -0.10 & & & 0.050 & & 0.140 & synt. \\
\hline $\mathrm{N}$ & 0.50 & & -0.10 & & & 0.350 & & 0.280 & synt. \\
\hline O I & $<0.40$ & & 0.20 & & 1 & -0.050 & & 0.250 & [OI] \\
\hline $\mathrm{Na} \mathrm{I}$ & 0.14 & 0.004 & -0.03 & 0.031 & 2 & -0.078 & 0.027 & 0.019 & \\
\hline Mg I & 0.35 & 0.119 & 0.35 & 0.039 & 3 & -0.250 & 0.081 & 0.047 & \\
\hline $\mathrm{Al}$ I & -0.38 & 0.091 & -0.21 & 0.079 & 2 & -0.418 & 0.013 & 0.009 & \\
\hline Si I & 0.23 & 0.106 & 0.13 & 0.050 & 11 & -0.149 & 0.067 & 0.020 & \\
\hline Si II & 0.18 & 0.011 & 0.09 & 0.029 & 2 & -0.162 & 0.018 & 0.013 & \\
\hline S I & 0.23 & 0.115 & 0.00 & 0.113 & 3 & -0.021 & 0.109 & 0.063 & \\
\hline $\mathrm{Ca} \mathrm{I}$ & 0.17 & 0.069 & 0.13 & 0.103 & 15 & -0.204 & 0.054 & 0.014 & \\
\hline Ca II & 0.16 & 0.129 & 0.15 & 0.183 & 3 & -0.239 & 0.068 & 0.039 & \\
\hline Sc II & 0.04 & 0.068 & 0.08 & 0.038 & 8 & -0.294 & 0.042 & 0.015 & \\
\hline Ti I & -0.02 & 0.050 & -0.07 & 0.066 & 17 & -0.201 & 0.032 & 0.008 & \\
\hline Ti II & 0.04 & 0.076 & 0.02 & 0.079 & 11 & -0.233 & 0.046 & 0.014 & \\
\hline V I & 0.18 & 0.280 & 0.02 & 0.139 & 10 & -0.080 & 0.344 & 0.109 & \\
\hline V II & 0.21 & 0.500 & 0.18 & 0.735 & 2 & -0.222 & 0.235 & 0.167 & \\
\hline $\mathrm{Cr}$ I & -0.10 & 0.091 & -0.06 & 0.100 & 29 & -0.297 & 0.065 & 0.012 & \\
\hline Cr II & -0.03 & 0.115 & 0.02 & 0.098 & 17 & -0.298 & 0.063 & 0.015 & \\
\hline Mn I & -0.20 & 0.009 & -0.27 & 0.029 & 3 & -0.181 & 0.030 & 0.017 & \\
\hline Co I & -0.31 & 0.151 & -0.15 & 0.166 & 3 & -0.404 & 0.024 & 0.014 & \\
\hline Ni I & -0.09 & 0.103 & -0.07 & 0.059 & 32 & -0.266 & 0.090 & 0.016 & \\
\hline $\mathrm{Cu} \mathrm{I}$ & -0.24 & 0.078 & -0.21 & 0.021 & 3 & -0.277 & 0.086 & 0.049 & \\
\hline Zn I & -0.01 & 0.041 & -0.11 & 0.158 & 3 & -0.150 & 0.124 & 0.072 & \\
\hline Sr I & -0.24 & & -0.36 & & 1 & -0.131 & & & \\
\hline Y II & -0.18 & 0.060 & -0.14 & 0.121 & 6 & -0.293 & 0.100 & 0.041 & \\
\hline $\mathrm{Zr} I$ & 0.09 & & 0.34 & & 1 & -0.500 & & & \\
\hline $\mathrm{Zr}$ II & -0.23 & & -0.46 & & 1 & -0.027 & & & \\
\hline Ba II & 0.12 & & 0.29 & & 1 & -0.413 & & & \\
\hline La II & -0.10 & & -0.13 & & 1 & -0.221 & & & \\
\hline Ce II & 0.08 & 0.009 & 0.21 & 0.085 & 3 & -0.380 & 0.093 & 0.053 & \\
\hline Nd II & 0.22 & 0.172 & 0.43 & 0.341 & 3 & -0.463 & 0.264 & 0.153 & \\
\hline
\end{tabular}

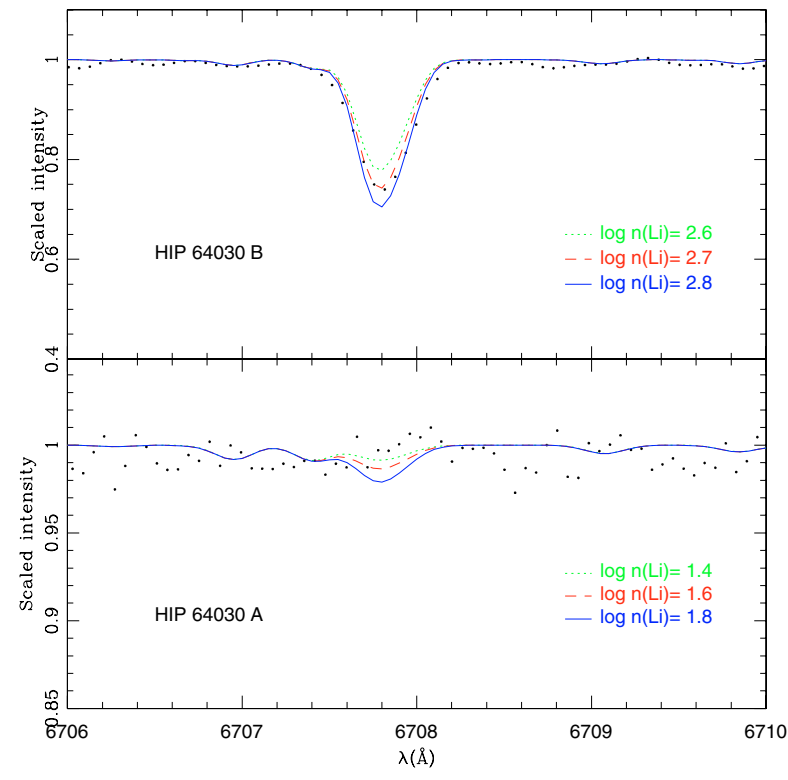

Fig. 7. Li spectral synthesis. Upper panel: HIP 64030B, lower panel: HIP 64030A.

material by the primary, but also of the large abundance difference of iron and most of the elements we measured (Table 6).



Fig. 8. Lithium abundance vs. effective temperature for the components of HIP 64030 (filled symbols) compared with those of stars in the Hyades (Thorburn et al. 1993; open circles: stars with Li detection; open squares: upper limits) and in M 67 (Jones et al. 1999; crosses: stars with Li detection; open triangles: upper limits).

Figure 9 shows the abundance difference as a function of condensation temperature, first ionization potential, and atomic number. A correlation with condensation temperature appears to be present; the abundance differences also show some 

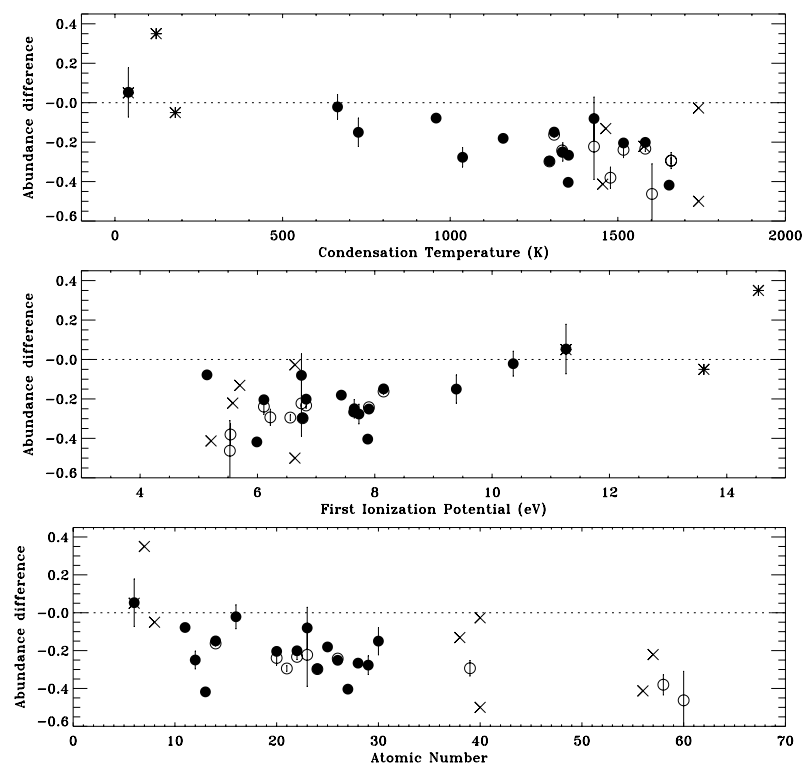

Fig. 9. Abundance difference as a function of condensation temperature. Filled and empty circles represent abundance differences from neutral and single-ionized species when at least two lines are available, while crosses represent elements for which one single line is included in the analysis. Asterisks represent the elements for which abundance was obtained by spectral synthesis.

correlation with first ionization potential, as the latter somewhat correlates with condensation temperature. However, the small abundance difference for sodium indicates that the real correlation is that with the condensation temperature.

Considering only elements with at least two measured lines, excluding $\mathrm{C}$ and $\mathrm{N}$ because of the alterations caused by the transfer of processed material from the RGB donor, and averaging the results from neutral and single ionized states when available (Fig. 10), the resulting linear correlation coefficient is -0.63 and the Spearman rank correlation coefficient is -0.56 with a significance of $98 \%$. Therefore, beside the alterations expected by the blue straggler formation process, the abundance difference between the components shows the pattern expected in the case of accretion of chemically fractionated material: no or low abundance difference for most of the elements characterized by a low condensation temperature, and large abundance difference for elements with high condensation temperature (e.g., iron) (Table 6; Fig. 10).

It must be noted that the condensation temperature of a given element is not an absolute value, but it depends on the physical conditions and global chemical composition of the gas. The adopted condensation temperatures are the "50\% condensation temperatures" (those at which half of the atoms of a given element are condensed and half are in gas phase) derived by Lodders (2003) for Solar System composition gas. The $\alpha$ enhancement, other chemical anomalies, and different physical conditions may alter the condensation sequence (Lodders \& Fegley 1995). From Fig. 10 there is some indication of peculiar features in the abundance difference vs. condensation temperature diagram: siderophile elements $(\mathrm{Fe}, \mathrm{Cu}, \mathrm{Cr}, \mathrm{Ni}, \mathrm{Co}$ ) have on average a larger abundance difference with respect to elements with similar condensation temperature condensing into oxygen compounds ( $\mathrm{Mg}, \mathrm{Si}, \mathrm{V}, \mathrm{Ca}, \mathrm{Ti})$. The differential analysis does not reveal peculiar differences of neutron-capture elements, confirming the results of relative abundances with respect to iron discussed above.

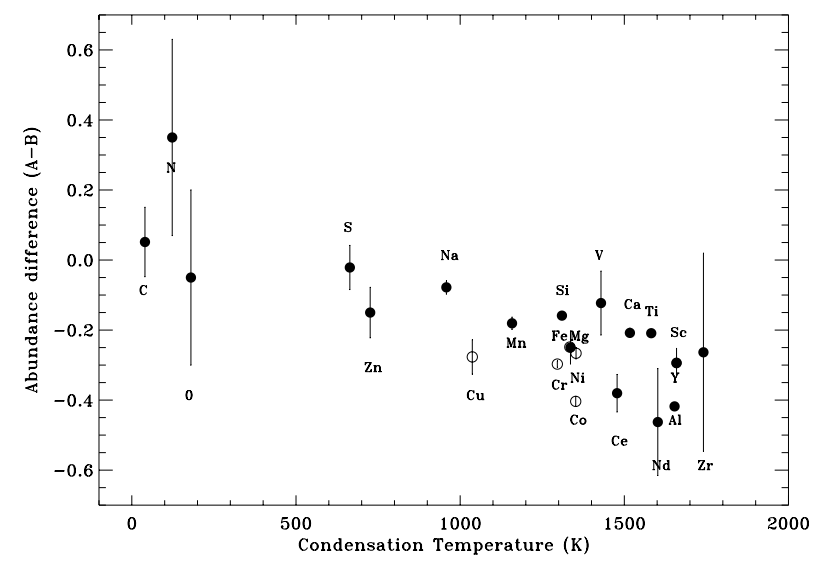

Fig. 10. Abundance difference as a function of condensation temperature. Only elements with at least two lines are plotted. Abundance difference from neutral and single-ionized species were averaged. Empty circles represent siderophiles elements, and filled circles the other elements. Siderophiles show a larger abundance difference with respect to other elements for similar condensation temperatures.

\section{Discussion}

The conclusion that can be drawn from the differential abundance analysis is that there is a real composition difference between the two components of HIP 64030, the primary, that is the blue stragglers, being more Fe poor by roughly a factor of two. The uniqueness of the large abundance difference of HIP 64030 among 50 pairs analyzed and its BSS status suggests a link between them. As discussed in Sect. 1, iron is not expected to be altered during the formation of the blue straggler. Abundance anomalies should be confined to light elements and, in the case of an AGB donor, on neutron capture elements. Therefore, while the signatures expected from mass transfer from a RGB (or lowmass AGB) star are indeed observed in the blue straggler component ( $\mathrm{N}$ enhancement, small ${ }^{12} \mathrm{C} /{ }^{13} \mathrm{C}$ isotopic ratio, no lithium), other mechanism(s) should play a fundamental role in the production of the observed abundance pattern.

We will now discuss two possible scenarios of selective accretion that may explain the origin of this abundance difference. Both of them envisage a similar evolution of the system. This is initially composed of a close binary (HIP 64030Aa and HIP 64030Ab) and of a third farther companion (HIP 64030B). Originally, the most massive of the stars is HIP 64030Ab, with a mass in the range between $0.9 M_{\odot}$ and $\sim 1.3-1.5 M_{\odot}$. The lower limit is the mass of HIP 64030B, and it is determined by the consideration that its evolution should be faster than that of HIP 64030B, which is only now evolving off of the main sequence. The upper limit is determined by the evolutionary age difference between HIP 64030Aa and HIP 64030B ( 5 Gyr), and by the consideration that if the mass transfer occurred too early, this second star would now be too evolved. The original mass of HIP 64030Aa is not well known; however, it should be smaller than the current mass of $1.04 M_{\odot}$. Hence the total mass of the system was originally lower than $\sim 3.3 M_{\odot}$.

When HIP 64030Ab evolved off of the main sequence, it filled its Roche lobe and began transferring significant amounts of material on HIP 64030Aa, as well as losing a considerable amount of mass (McCrea channel of formation of the blue stragglers). Notice that the mass transfer episode probably occurred during the first ascent of the RGB since there is no trace of triple alpha reaction or s-process products in the composition of HIP 64030Aa. Alternatively, the mass transfer episode 
can also have occurred during the AGB phase of a star not massive enough to produce significant s-element enhancements. Mass transfer ended when the outer convective envelope of HIP 64030Ab was entirely consumed, leaving only the degenerate core, which is probably a He-white dwarf of $\sim 0.2-0.4 M_{\odot}$. HIP 64030Aa acquired material from the convective envelope of HIP 64030Ab: a lower limit to the accreted mass is given by the mass difference with HIP $64030 \mathrm{~B}$, that is about $0.1 M_{\odot}$. This is much more than the mass of its convective envelope, so that we may assume hereinafter that its convective envelope is entirely composed of material originally in the convective envelope of HIP 64030Aa. This agrees well with its chemical abundance pattern.

The orbit of the binary HIP 64030A-B should have widened considerably during the mass loss episode. The details of the orbital evolution depend critically on the comparison between the timescale of the mass ejection and the orbital period. The mass loss by stellar wind before Roche lobe overflow should have caused a small adiabatic widening of the orbit $\left(a_{\text {end }}=\right.$ $\left.a_{\text {start }} * m_{\text {start }} / m_{\text {end }}\right)$, without significant changes to the orbital eccentricity. For the mass loss during the common envelope phase, the situation is different, as hydrodynamical simulations (e.g., Sandquist et al. 1998; Rasio \& Livio 2000) indicate a very rapid ejection ( $\sim$ years), much shorter than the orbital period of the wide component. In this case, the widening of the orbit can be much larger (Boersma 1961). In our case, the system remains bound, as the final mass of the system is $\sim 2.1-2.4 M_{\odot}$, well above half of the original mass. If a major part of mass loss from the central binary occurred during the common envelope phase, we may reasonably guess that the original separation between HIP 64030A and B was about one half or one third of the present one. Considering the current projected separation of $460 \mathrm{AU}$, and the orbits compatible with the observations (Sect. 3.2), the original binary separation may then have been about 100-200 AU. Closer original separations are possible if the common envelope ejection occurred by chance close to the periastron passage of the wide companion, as in this case the widening of the orbit can be much larger (Hills 1983).

\subsection{Scenario A. Selective accretion of metal-depleted material on the primary}

The basic idea of this scenario is that HIP 64030B is a completely normal star, and its surface composition is identical to the original system composition. All anomalies are then confined to HIP 64030Aa. In this case there is a depletion of the original Fe content at the surface of HIP 64030Aa.

To explain the anomalous Fe-peak content of HIP 64030Aa, the idea is to call for a mechanism similar to that found acting in post-AGB binaries (Van Winckel 2003). Large Fe depletions (by several orders of magnitude) have been observed in these stars. The mechanism called for envisages the formation of a circumbinary torus, from which dust is selectively removed by radiation pressure and high velocity wind. Part of the remaining (dust-free) gas is then accreted again by the central star (Waters et al. 1992). In the case of post-AGB stars, the amount of gas accreted by the star need not be large because there is not any convective envelope. In the case of HIP 64030Aa, this should be comparable to the mass $\left(\leq 0.01 M_{\odot}\right)$ of the outer convective envelope, to cause enough dilution effect for Fe.

The $R V$ Tauri and post AGB with selective depletion are mostly and likely all binaries (Van Winckel et al. 1999). Their orbital periods and eccentricity overlap with those of field blue stragglers (Fig. 11). The accretion of chemically fractionated

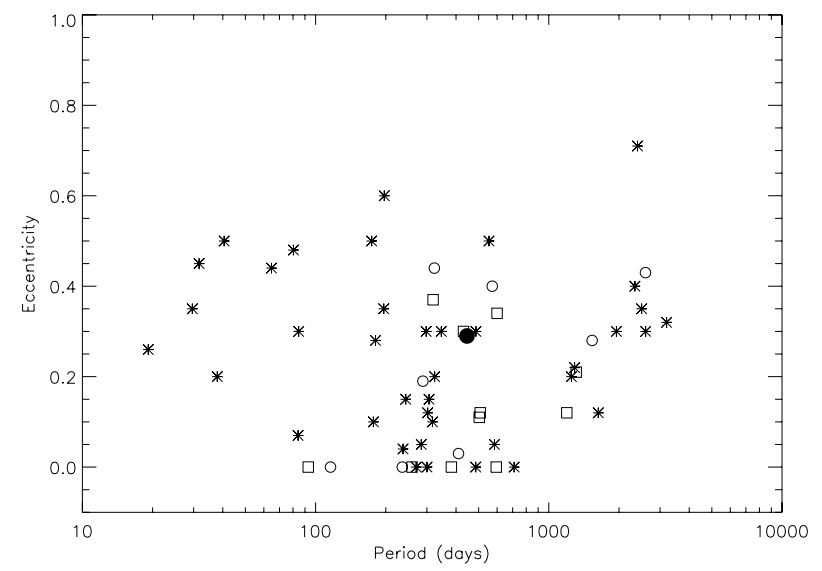

Fig. 11. Period eccentricity relation for post AGB and $R V$ Tauri stars with depleted abundances (empty squares) and normal abundances (empty circles) from Maas (2003) and of field blue stragglers studied by Preston \& Sneden (2000) and Carney et al. (2005) (asterisks). The position of HIP 64030 is overplotted as a filled circle.

material observed on the post-AGB component should also occur on the companion. Such a companion, if the amount material received during the mass transfer event is large enough, will become a blue straggler, while the ex-post AGB component will become a faint white dwarf. A possible case of a future blue straggler among the companion of post-AGB binaries is represented by the companion of IRAS 05208-2035 (Maas 2003).

While Keplerian disks appear to be common around postAGB binaries (De Ruyter et al. 2006), it is not clear if a significant fraction of the heavy material may really condense onto dust in a torus around an RGB star (probably the case of HIP 64030A). If this mechanism is really active for HIP 64030, it should be so in other blue stragglers formed with the McCrea mechanism. However, we are not aware of other evidence for chemically fractionated accretion on blue stragglers. Blue stragglers observed in M 67 (Shetrone \& Sandquist 2000) share the same Fe content of the other cluster star (one case with lower metal abundance is probably due to the composite nature of the spectrum). For field blue stragglers, it is not easy to identify anomalies in element abundances and abundance ratios at the level of 0.2 dex. Anyway, available literature data for field blue stragglers are quite sparse. Most of abundance analysis of these stars do not include key elements to test the accretion of chemically fractionated material (e.g., sodium, sulfur, zinc), focusing their attention mostly on the search for anomalies of light elements and neutron capture elements (e.g., Sneden et al. 2003). It should also be considered that the gas-dust separation might be at work only above a metallicity threshold (e.g., $[\mathrm{Fe} / \mathrm{H}]=-1.0$, as suggested by Giridhar et al. 2000 for $R V$ Tauri stars). If this is the case, the pattern should not be observed in the most metal poor blue stragglers. Finally, this mechanism does not explain the unusual ${ }^{12} \mathrm{C} /{ }^{13} \mathrm{C}$ isotopic ratio found for HIP 64030B in a natural way. We are then forced to suggest an independent (primordial) origin for it (the high lithium content of the secondary allows us to rule out the onset of first dredge-up for this star).

\subsection{Scenario B. Selective accretion of metal-rich material on the secondary}

The basic idea of this scenario is that HIP 64030Aa has a normal abundance pattern for its evolutionary status, and that the abundance difference found between the two visible components is 
due to a combination of the classical evolutionary pattern of a blue straggler, and of selective accretion on HIP 64030B. In this scheme, the original Fe-peak element abundances of the system is that found in HIP 64030Aa.

There are two basic assumptions in this scenario:

- HIP 64030B should have been able to capture a large enough fraction of the material expelled from the primary (which is of the order of $1 M_{\odot}$ ). This should be at least comparable to the present mass of its convective envelope ( 0.015-0.030 $M_{\odot}$ ) because it should include an amount of Fe large enough to roughly double its original Fe abundance.

- There should have been some selective accretion mechanism. The basic idea here is that the accreting material might have formed a disk around HIP 64030B, similar to a protoplanetary disk. On a short timescale, dust settled at the equator of this disk. Then, the same mechanism called for planet migration may have been onset, causing infall of rocky material on the central star. Since when this episode occurred HIP 64030B was already a well evolved, old star, the convective envelope was already in place when this disk evolved, so that quite small amounts of rocky material (a few Earth masses) would be enough to significantly enrich the surface of the star.

To evaluate the plausibility of the occurrence of an adequate amount of accretion on HIP 64030B (about $0.01 M_{\odot}$ ), we first considered the prescriptions of the Bondi-Hoyle wind accretion (see, e.g., Hurley et al. 2002). It results that for original masses of the inner pair $\sim 1.5 M_{\odot}$ and $\sim 0.9 M_{\odot}$, a binary separation as small as 20-30 AU is required, while at $100 \mathrm{AU}$ the accreted mass should be of the order of $0.001 M_{\odot}$. Hydrodynamical simulations by Theuns et al. (1996) found lower accretion rates with respect to the standard Bondi-Hoyle ones, when the wind speed is comparable to orbital velocity, which occurs for separation smaller than about $20 \mathrm{AU}$. It must be noted that these simulations refer to the accretion of mass lost by a single star through stellar wind, while we expect that the blue straggler HIP 64030A evolved through Roche lobe overflow and common envelope phase. The velocity, time and spatial distribution, and other properties of the material lost by the inner binary should be different with respect to the stellar wind from an isolated star, possibly significantly affecting the amount of accreted material by a wide companion. The available models of the ejection of the common envelope show large spatial inhomogeneities in the outflow, with most of the material ejected in the orbital plane of the close binary (see, e.g., Sandquist et al. 1998). Therefore, a suitable orientation of the orbital planes of the triple system might provide larger accretion rates, if the ejection velocity is similar to that of stellar wind. Further studies are required to evaluate the plausibility of this scenario.

As possible pros of this scenario, we notice that with respect to Scenario A, in this scenario we may invoke the same selective accretion mechanism to explain the unusual ${ }^{12} \mathrm{C} /{ }^{13} \mathrm{C}$ isotopic ratio found for HIP 64030B. On the other hand, it might require some fine tuning in order for enough matter to be accreted by HIP 64030B. The rather high lithium content of HIP 64030B is not naturally explained by this scenario, as the material falling into the star should have been lithium-depleted. The validity of this scenario can be tested by looking for abundance anomalies of wide companions orbiting blue stragglers, other stars on which mass transfer from an evolved companion occurred (e.g., barium stars, $\mathrm{CH}$ stars), and central stars of planetary nebulae.

\section{Summary}

The system HIP 64030 is composed of two moderately metalpoor solar-mass stars with a projected separation of about $500 \mathrm{AU}$, with the primary being a spectroscopic binary with a period of 445 days and a companion mass larger than $0.17 M_{\odot}$. The (currently) most massive component appears to be a blue straggler. High-precision differential abundance analysis reveals the signatures of the mass transfer expected for a blue straggler formed via McCrea mechanism, but also an unexpected difference of most of the other elements, including iron, correlated with the condensation temperature.

Two scenarios were devised to explain the observed abundance pattern. In the first one, all the abundance anomalies occur on the blue straggler. Its lower metal abundance would be explained by the accretion of dust-depleted material, in a similar way to what has occurred for several post-AGB binaries. The second scenario is based on the accretion of dust-rich material on the secondary. It may at least qualitatively explain the whole abundance pattern (including the low ${ }^{12} \mathrm{C} /{ }^{13} \mathrm{C}$ of the secondary, unexpected for a normal main sequence star). However, it requires that a rather large amount of material lost by the blue straggler system be captured by the secondary. Whether this is actually realistic would require a dedicated modeling. A refinement of the constraints available for the binary orbit (new highprecision relative astrometric observations, improvement on distance estimate) would also make the evaluation of this scenario easier.

The reality of either scenario implies testable predictions. For the first scenario, alterations of iron abundance and trends of abundance with condensation temperature should be observed in other blue straggler formed via mass transfer. For the second scenario, signature of accretion of chemically processed material should be found for wide companions of blue stragglers and other related objects.

Acknowledgements. This research has made use of the SIMBAD database, operated at CDS, Strasbourg, France. We warmly thank R. Cosentino, A. Martinez Fiorenzano, R. Wittenmyer, and W. Cochran for the observations at TNG and Mc Donald Observatory. We thank M. Barbieri for the computation of the galactic orbit. We ackowledge throughful comments by the referee Dr. Preston. This work was partially funded by COFIN 2004 "From stars to planets: accretion, disk evolution, and planet formation" by Ministero Università e Ricerca Scientifica Italy. M. Endl is supported by the National Aeronautics and Space Administration under grants NNG 04-G141G and NNG 05-G107G.

\section{References}

Abt, H. A. 1981, ApJS, 45, 437

Aitken, R. G. 1932, New general catalogue of double stars within 120 of the North pole, Carnegie Inst. Washington Pub., No. 417

Alzner, A. 1998, A\&AS, 132, 237

Andersen, J., \& Nordstrom, B. 1985, A\&AS, 62, 355

Barbieri, M., \& Gratton, R. 2002, A\&A, 384, 879

Boersma, J. 1961, Bull. Astron. Inst. Netherl., 15, 291

Carney, B. W., Latham, D. W., \& Laird, J. B. 2005, AJ, 129, 466

Cody, A. M., \& Sasselov, D. D. 2005, ApJ, 622, 704

Cutri, R. M., Skrutskie, M. F., Van Dyk, S., et al. 2003, Explanatory Supplement to the 2MASS All Sky Data Release, www.ipac. caltech.edu/2mass

Desidera, S., Gratton, R. G., Endl, M., et al. 2003, A\&A, 405, 207

Desidera, S., Gratton, R. G., Scuderi, S., et al. 2004, A\&A, 420, 683

Desidera, S., Gratton, R. G., Claudi, R. U., et al. 2006a, Proc. conference Tenth Anniversary of 51 Peg-b: status of and prospects for hot Jupiter studies, 119

Desidera, S., Gratton, R. G., Lucatello, S., Claudi, R. U., \& Dall, Th. 2006b, A\&A, 454, 553

Desidera, S., Gratton, R. G., Lucatello, S., \& Claudi, R. U. 2006c, A\&A, 454, 581

De Ruyter, S., Van Winckel, H., Maas, T., et al. 2006, A\&A, 448, 641

Duquennoy, A., \& Mayor, M. 1991, A\&A, 248, 485

Endl, M., Kürster, M., \& Els, S. 2000, A\&A, 363, 585 
ESA 1997, The Hipparcos and Tycho Catalogues, ESA SP-1200

Fuhrmann, K., \& Bernkopf, J. 1999, A\&A, 347, 897

Gasteyer, C., \& Eichhorn, H. 1964, AJ, 69, 762

Giridhar, S., Lambert, D. L., \& Gonzalez, G. 2000, ApJ, 531, 521

Gratton, R. G., Sneden, C., Carretta, E., \& Bragaglia, A. 2000, A\&A, 354, 169

Gratton, R. G., Bonanno, G., Bruno, P., et al. 2001, Exp. Astron., 12, 107

Gratton, R. G., Carretta, E., Claudi, R., Lucatello, S., \& Barbieri, M. 2003, A\&A, 404, 187

Hauser, H., \& Marcy, G. W. 1999, PASP, 111, 321

Heintz, W. D. 1980, ApJS, 44, 111

Hills, J. G. 1983, ApJ, 267, 322

Hurley, J. R., Tout, C. A., \& Pols, O. R. 2002, MNRAS, 329, 897

Jones, B. F., Fischer, D., \& Soderblom, D. R. 1999, AJ, 117, 330

Lodders, K. 2003, ApJ, 591, 1220

Lodders, K., \& Fegley, B. 1995, Meteoritics, 30, 661

Lucatello, S., Gratton, R., Cohen, J. G., et al. 2003, AJ, 125, 875

Maas, T. 2003, Ph.D. Thesis

McClure, R. D. 1997, PASP, 109, 536

McCrea, W. H. 1964, MNRAS, 128, 147

Muller, P. 1950, J. Obs., 33, 105

Nordstrom, B., Mayor, M., Andersen, J., et al. 2004, A\&A, 418, 989

Norris, J. E., Ryan, S. G., Beers, T. C., \& Delyannis, C. P. 1997, ApJ, 485, 370

Olsen, E. H. 1998, A\&AS, 129, 431

Pannunzio, R., \& Morbidelli, R. 1983, A\&AS, 54, 489

Preston, G. W., \& Sneden, C. 2000, AJ, 120, 1014
Rasio, F., \& Livio, M. 1996, ApJ, 471, 366

Ryan, S. G., Beers, T. C., Kajino, T., \& Rosolankova, K. 2001, ApJ, 547, 231

Ryan, S. G., Gregory, S. G., Kolb, U., Beers, T. C., \& Kajino, T. 2002, ApJ, 571, 501

Salasnich, B., Girardi, L., Weiss, A., \& Chiosi, C. 2000, A\&A, 361, 1023

Sandquist, E. L., Taam, R. E., Chem, X., Bodenheimer, P., \& Burkert, A. 1998, ApJ, 500, 909

Shetrone, M. D., \& Sandquist, E. L. 2000, AJ, 120, 1913

Sneden, C., Preston, G., \& Cowan, J. J. 2003, ApJ, 592, 504

Söderhjelm, S. 1999, A\&A, 341, 121

Struve, O., \& Zebergs, V. 1959, AJ, 64, 219

Theuns, T., Boffin, H. M. J., \& Jorissen, A. 1996, MNRAS, 280, 1264

Thorburn, J. A., Hobbs, L. M., Deliyannis, C. P., \& Pinsonneault, M. H. 1993, ApJ, 415, 150

Van Dessel, E., \& Sinachopoulos, D. 1993, A\&AS, 100, 517

Van Winckel, H. 2003, ARA\&A, 41, 391

Van Winckel, H., Waelkens, C., Fernie, J. D., \& Waters, L. B. F. M. 1999, A\&A, 343,202

Voges, W., Aschenbach, B., Boller, Th., et al., ROSAT All-Sky Survey Faint source Catalogue

Waters, L. B. F. M., Trams, N. R., \& Waelkens, C. 1992, A\&A, 262, L37

Wilson, R. E. 1953, General Catalogue of Stellar Radial Velocities Carnegie Inst. Washington D.C. Publ., 601

Wittenmyer, R. A., Endl, M., Cochran, W. D., et al. 2006, AJ, 132, 177 